\title{
The second generation of HIV-1 vertically exposed infants: a case series from the Italian Register for paediatric HIV infection
}

\author{
Carmelina Calitri ${ }^{1}$, Clara Gabiano ${ }^{1}$, Luisa Galli², Elena Chiappini ${ }^{2}$, Carlo Giaquinto ${ }^{3}$, Wilma Buffolano ${ }^{4}$, \\ Orazio Genovese ${ }^{5}$, Susanna Esposito ${ }^{6}$, Stefania Bernardi ${ }^{7}$, Maurizio De Martino ${ }^{2}$, Pier-Angelo Tovo ${ }^{1 *}$, for the \\ Italian Register for HIV Infection in Children
}

\begin{abstract}
Background: In the Highly Active Antiretroviral Therapy (HAART) era, the prognosis of children perinatally infected with HIV-1 has significantly improved, so the number of perinatally-infected females entering child-bearing age and experiencing motherhood is increasing.

Methods: A description of the medical history and pregnancy outcomes of women with perinatal acquired HIV-1 infection enrolled in the Italian Register for HIV infection in Children.

Results: Twenty-three women had 29 pregnancies. They had started an antiretroviral therapy at a median of 7.7 years (interquartile range, IQR 2.3 - 11.4), and had experienced a median of 4 therapeutic regimens (IQR 2-6). Twenty women (87\%) had taken zidovudine (AZT) before pregnancy, in 14 cases as a starting monotherapy. In 21 pregnancies a protease inhibitor-based regimen was used. At delivery, the median of CD4+ T lymphocytes was 450/ML (IQR 275-522), and no viral load was detectable in 15 cases (reported in 21 pregnancies). Twenty-eight children were delivered through caesarean section (median gestational age: 38 weeks, IQR 36-38, median birth weight: 2550 grams, IQR 2270 - 3000). Intravenous AZT was administered during delivery in 26 cases. All children received oral AZT (median: 42 days, IQR 31 - 42), with no adverse events reported. No child acquired HIV-1 infection.
\end{abstract}

Conclusions: Despite a long history of maternal infection, multiple antiretroviral regimens and, perhaps, the development of drug-resistant viruses, the risk of mother-to-child transmission does not seem to have increased among the second-generation of HIV-1 exposed infants.

Keywords: HIV-1, Drug-resistant virus, AZT, Vertical transmission

\section{Background}

The introduction of Highly Active Antiretroviral Therapy (HAART) has significantly improved the quality of life and prognosis of children with perinatally acquired HIV-1 infection in resource-rich countries [1,2]. As a result, an increasing number of these children are entering adolescence and young adulthood, with a consequent proportion of females becoming sexually active and pregnant. These mothers presented with a long history of HIV-1 infection and sometimes AIDS complications. They had a wide exposition to antiretroviral (ART) drugs

\footnotetext{
*Correspondence: pierangelo.tovo@unito.it

'Department of Paediatrics, University of Turin, Turin, Italy

Full list of author information is available at the end of the article
}

and to their, primarily metabolic, chronic side effects. They experienced multiple ART regimens, which were often sub-therapeutic until the advent of HAART, with a consequent risk of acquiring drug-resistant viruses.

Therefore, it has since become crucial to define the pregnancy outcome and effectiveness of ART therapy in preventing mother-to-child HIV-1 transmission (MTCT) in this 2nd generation of HIV-1 exposed infants. Reports from Europe [3,4], Puerto Rico [5], India [6], USA [7-10], and Brazil [11] describe favourable maternal and neonatal outcomes, with a MTCT rate varying from 0 to $7.7 \%$, detected in cases of poor adherence to therapy [10].

Here, we describe the clinical aspects of pregnancy in women with perinatally acquired HIV-1 infection enrolled 
over the years in the Italian Register for HIV Infection in Children.

\section{Methods}

The Italian Register for HIV infection in children (ITLR) is a nationwide, multicentre, prospective study set up by the Italian Association of Paediatrics in 1985. At the moment of patient's enrolment, written informed consent is obtained from the patient's guardians; data are treated anonymously as each patient is identified by an alphanumerical code. The study was approved by the review boards and ethics committees of each participating institution, as described in detail elsewhere $[1,12]$. A retrospective analysis of perinatally HIV-1 infected females, enrolled in the ITLR from its institution and who had a child, was carried out. Information included: the women's demographic and clinical characteristics, such as their mothers' risk factors for HIV-1 infection, perinatal ART use, HIV-1 related diseases and comorbidities; the number and types of ART regimens both before and during pregnancy; age at delivery, number of CD4+ Tlymphocytes and viral load at delivery, the mode of delivery; infants' characteristics (gestational age, sex, birth weight, congenital malformations, any other acute or chronic diseases, adverse events for MTCT prophylaxis, HIV-1 infection status). SPSS (version 20.0) software for Windows was used for data management.

\section{Results}

Between 2001 and 2012, 29 children were born to 23 women with perinatal HIV-1 infection. One woman had 3 pregnancies and four women had 2 pregnancies each. See Tables 1, 2 and 3 for data concerning mother-child pairs.

The median maternal age at first delivery was 22 years (interquartile range (IQR) 20.1-24.3); the oldest mother was born in 1982, the youngest in 1991. No infected mother was perinatally exposed to antiretroviral drugs, as ART prophylaxis was not used at that time. The grandmothers' risk factors for HIV-1 infection were known for 17 women: 11 had had high risk sexual intercourse and 6 had been IV drug users. All but one woman had ART therapy during childhood or adolescence. The median age at the start of ART therapy was 7.7 years (IQR 2.3-11.4). The median number of regimens for each woman was 4 (IQR 2-6). Twenty (87\%) women were given zidovudine (AZT), in 14 cases as a starting monotherapy for a median period of 4.4 years (IQR 2.0-5.2); 12/14 patients were later shifted to a dual therapy containing AZT (median period 2.4 years, IQR 1.0-3.4). All but 2 women received HAART before pregnancy, with a combination of 3 or more drugs, containing at least one protease-inhibitor (PI) in 16 cases. The median time of the mothers' exposure to ART drugs before their first pregnancy was 11.6 years (IQR 9.5-17.5). Four women developed an AIDS defining condition (at a median age of 9.0 years, IQR 1.8-12.7); 10 women were classified, according to the CDC paediatric classification, in clinical category B, 8 in category A, 1 in category N. As far as the immunological status is concerned, 5 were in category 1,8 in category 2 , and 10 in category 3 .

Ten mothers did not have any ART therapy at the time of conception; of the ten, 2 continued without therapy throughout their pregnancy, 4 started ART therapy in the first trimester and 4 after the $12^{\text {th }}$ week of gestation, including one woman who started a rescue therapy including the integrase inhibitor raltegravir at the $38^{\text {th }}$ week of gestation. In the other pregnancies, the women were already undergoing ART treatment at conception, and 3 of them discontinued therapy in the first trimester (see Table 2). In one case, efavirenz (EFV) was continued during the first 5 weeks of gestation, with a switch to a lopinavir/ritonavir containing combination after the $12^{\text {th }}$ week. A PI-based regimen was used in a total of 21 pregnancies. No complications related to pregnancy were noticed. There was a median of $450 / \mu \mathrm{L}$ (IQR 275-522) CD4+ T lymphocytes at the last check before delivery (reported in 23 of the 29 pregnancies); all women with a previous AIDS-defining condition had $>200 \mathrm{CD} 4+/ \mu \mathrm{L}$. The viral load at delivery (reported in 21 pregnancies) was undetectable in 15 cases. Intravenous AZT was administered during 26 (89.6\%) deliveries. Twenty-eight children were born through caesarean section, which was elective in 26 cases. No neonatal complications for children at birth were reported; 17 (58.6\%) were females; their median gestational age was 38 weeks (IQR 36-38) and the median birth weight was 2550 grams (IQR $2270-3000)$. Eight were preterm $(<37$ weeks of gestation), and 4 were small for the gestational age (SGA) (Table 3) [13]. No infant was breastfed. Twenty-six infants received oral AZT as MTCT prophylaxis; 2 brothers received a combined regimen $(\mathrm{AZT}+3 \mathrm{TC}+\mathrm{NVP}$ single dose): the first child because of lack of maternal ART therapy during pregnancy, the second one because of severe maternal immunosuppression (CD4+ Tlymphocytes at labour: 95 cell/ $\mu \mathrm{L}$ ). Neonatal ART prophylaxis was administered for a median period of 42 days (IQR 31-42) with no major adverse events.

No child acquired HIV-1 infection. One patient was HCV infected and one presented cryptorchidism. The median age at the last check was 3 years (IQR 1.3-4.3), with nine children followed over 4 years of age.

\section{Discussion}

There are few studies exploring the pregnancy outcome in HIV-1 perinatally-infected women. Despite the limited number of pregnancies enrolled and limited amount of available data, ours is one of the largest cohorts with detailed information on the entire maternal history.

The results are reassuring, since none of the 29 exposed infants acquired the infection. Indeed, of the 134 
Table 1 Characteristics of HIV-1 vertically infected women

\begin{tabular}{|c|c|c|c|c|c|c|c|c|c|c|}
\hline N. & $\begin{array}{l}\text { Birth } \\
\text { order }\end{array}$ & $\begin{array}{l}\text { Risk factors for HIV } \\
\text { infection in grandmothers }\end{array}$ & $\begin{array}{l}\text { Year of } \\
\text { birth }\end{array}$ & $\begin{array}{l}\text { Age (years) at } \\
\text { start of ART } \\
\text { therapy }\end{array}$ & $\begin{array}{c}\text { AZT } \\
\text { monotherapy }\end{array}$ & $\begin{array}{c}\text { No of } \\
\text { regimens } \\
\text { experienced }\end{array}$ & $\begin{array}{l}\text { Years of ART } \\
\text { therapy (before the } \\
\text { first pregnancy) }\end{array}$ & $\begin{array}{l}\text { Clinical and } \\
\text { immunological } \\
\text { category at last } \\
\text { observation }\end{array}$ & $\begin{array}{l}\text { Age (years) } \\
\text { at AIDS onset }\end{array}$ & $\begin{array}{l}\text { Age (years) } \\
\text { at delivery }\end{array}$ \\
\hline Case 1 & $1^{\text {st }}$ & Drug abuse & 1982 & 14.6 & No & 4 & 13.2 & A 1 & & 28.4 \\
\hline \multirow[t]{2}{*}{ Case 2} & $1^{\text {st }}$ & Sexual intercourse with drug abuser, Drug abuse & 1982 & 10.6 & Yes & 5 & 7.7 & B 3 & & 24.6 \\
\hline & $2^{\text {nd }}$ & & & & & & & & & 26.9 \\
\hline Case 3 & $1^{\text {st }}$ & Risk sexual intercourse & 1983 & 7.7 & Yes & 6 & 17.5 & B 3 & & 26.1 \\
\hline Case 4 & $1^{\text {st }}$ & Drug abuse & 1983 & 11.4 & Yes & 7 & 7 & B 3 & & 23.9 \\
\hline \multirow[t]{3}{*}{ Case 5} & $1^{\text {st }}$ & Unknown & 1983 & 21.7 & No & 1 & 0 & B 3 & & 22 \\
\hline & $2^{\text {nd }}$ & & & & & & & & & 23.7 \\
\hline & $3^{\text {th }}$ & & & & & & & & & 24.7 \\
\hline \multirow[t]{2}{*}{ Case 6} & $15 t$ & Drug abuse & 1984 & 6.6 & Yes & 6 & 9.7 & B 2 & & 17 \\
\hline & $2^{\text {nd }}$ & & & & & & & & & 18.3 \\
\hline Case 7 & $1^{\text {st }}$ & Sexual intercourse with drug abuser & 1985 & 3.1 & No & 2 & 20.5 & N 2 & & 24.3 \\
\hline Case 8 & $1^{\text {st }}$ & Drug abuse & 1985 & 3 & Yes & 15 & 17.7 & $C 3$ & 11.5 & 22.4 \\
\hline Case 9 & $1^{\text {st }}$ & $\begin{array}{c}\text { Sexual intercourse with drug abuser, Drug } \\
\text { abuse }\end{array}$ & 1986 & 12.2 & No & 2 & 9.5 & $\mathrm{C} 2$ & 13.1 & 22.2 \\
\hline Case 10 & $1^{\text {st }}$ & Sexual intercourse with drug abuser & 1986 & 6.6 & Yes & 6 & 14.8 & C 3 & 6.6 & 22.1 \\
\hline Case 11 & 1 st & Unknown & 1986 & 10.2 & No & 6 & 11.1 & A 3 & & 22 \\
\hline \multirow[t]{2}{*}{ Case 12} & $1^{\text {st }}$ & Sexual intercourse with drug abuser & 1986 & 10 & Yes & 3 & 6.3 & A 2 & & 21.1 \\
\hline & $2^{\text {nd }}$ & & & & & & & & & 22.2 \\
\hline Case 13 & $1^{\text {st }}$ & Unknown & 1986 & 13.3 & No & 1 & 11 & A 1 & & 25 \\
\hline Case 14 & $1^{\text {st }}$ & Sexual intercourse with drug abuser & 1987 & 2.3 & Yes & 3 & 21.6 & B 2 & & 24.5 \\
\hline Case 15 & $15 t$ & Unknown & 1988 & 14.4 & No & 2 & 10.3 & B 1 & & 22.6 \\
\hline Case 16 & $1^{\text {st }}$ & Unknown & 1988 & 1.9 & Yes & 2 & 6.2 & A 2 & & 19.2 \\
\hline Case 17 & $1^{\text {st }}$ & Sexual intercourse with drug abuser & 1989 & 1.8 & Yes & 5 & 18 & A 1 & & 20.3 \\
\hline Case 18 & $1^{\text {st }}$ & Sexual intercourse with drug abuser, Drug abuse & 1990 & 1.7 & Yes & 5 & 14.4 & B 2 & & 20.1 \\
\hline Case 19 & $1^{\text {st }}$ & Drug abuse & 1990 & 5.4 & Yes & 8 & 13.9 & B 3 & & 20 \\
\hline \multirow[t]{2}{*}{ Case 20} & 1st & Unknown & 1990 & 8.6 & No & 4 & 11.6 & A 3 & & 20.8 \\
\hline & $2^{\text {nd }}$ & & & & & & & & & 21.7 \\
\hline Case 21 & $1^{\text {st }}$ & Sexual intercourse with drug abuser & 1991 & 1.2 & Yes & 3 & 17.3 & B 1 & & 19.3 \\
\hline Case 22 & $1^{\text {st }}$ & Drug abuse & 1991 & 0.6 & Yes & 7 & 19.2 & $C 3$ & 0.2 & 20.4 \\
\hline Case 23 & $1^{\text {st }}$ & Sexual intercourse with drug abuser, Drug abuse & 1991 & 8.2 & No & 2 & 9.9 & A 2 & & 20 \\
\hline
\end{tabular}

Legend. ART = antiretroviral; $A Z T$ = zidovudine. 


\section{Table 2 Pregnancy features}

\begin{tabular}{|c|c|c|c|c|c|c|c|c|c|c|}
\hline N. & $\begin{array}{l}\text { Birth } \\
\text { order }\end{array}$ & $\begin{array}{l}\text { ART therapy } \\
\text { at conception }\end{array}$ & $\begin{array}{l}\text { ART therapy interruption } \\
\text { at first trimester }\end{array}$ & ART regimen during pregnancy & $\begin{array}{l}\text { Gestational } \\
\text { week at start }\end{array}$ & $\begin{array}{l}\text { Gestational } \\
\text { week at end }\end{array}$ & CD4+ at delivery & VL at delivery & AZT intrapartum & $\begin{array}{l}\text { Type of } \\
\text { delivery }\end{array}$ \\
\hline Case 1 & $1^{\text {st }}$ & No & No & $\mathrm{AZT}+3 \mathrm{TC}+\mathrm{ATV} / \mathrm{rtV}$ & 5 & 37 & 525 & Undetectable & Yes & EC \\
\hline \multirow[t]{3}{*}{ Case 2} & $1^{\text {st }}$ & Yes & Yes (from $5^{\text {th }}$ to $12^{\text {th }}$ week) & $\mathrm{DDI}+3 \mathrm{TC}+\mathrm{NVP}$ & 1 & 4 & 480 & 157 & Yes & EC \\
\hline & & & & $A Z T+3 T C+N V P$ & 13 & 38 & & & & \\
\hline & $2^{\text {nd }}$ & Yes & No & $\mathrm{FTC}+\mathrm{TDF}+\mathrm{FPV} / \mathrm{rtv}$ & 1 & 38 & 520 & Undetectable & Yes & EC \\
\hline Case 3 & $1^{\text {st }}$ & Yes & No & $\mathrm{FTC}+\mathrm{TDF}+\mathrm{ATV} / \mathrm{rtV}$ & 1 & 38 & 125 & Undetectable & Yes & EC \\
\hline Case 4 & $1^{\text {st }}$ & No & No & No & & & Unknown & Unknown & Yes & EC \\
\hline \multirow[t]{3}{*}{ Case 5} & $1^{\text {st }}$ & No & No & $\mathrm{AZT}+3 \mathrm{TC}+\mathrm{LPV} / \mathrm{rtV}$ & 23 & 34 & 307 & Unknown & Yes & EC \\
\hline & $2^{\text {nd }}$ & Yes & No & $\mathrm{AZT}+3 \mathrm{TC}+\mathrm{LPV} / \mathrm{rtV}$ & 1 & 37 & 480 & 15332 & Yes & EC \\
\hline & $3^{\text {th }}$ & Yes & No & $\mathrm{AZT}+3 \mathrm{TC}+\mathrm{LPV} / \mathrm{rtV}$ & 1 & Unknown & Unknown & Unknown & Yes & EC \\
\hline \multirow[t]{2}{*}{ Case 6} & $1^{\text {st }}$ & No & No & $A Z T+3 T C+N V P$ & 6 & 36 & Unknown & Unknown & No & EC \\
\hline & $2^{\text {nd }}$ & No & No & $A Z T+3 T C+N V P$ & 6 & 40 & Unknown & Unknown & No & EC \\
\hline \multirow[t]{2}{*}{ Case 7} & $1^{\text {st }}$ & Yes & No & $A B C+3 T C+A T V$ & 1 & 34 & 323 & Undetectable & Yes & EC \\
\hline & & & & $\mathrm{ABC}+3 \mathrm{TC}+\mathrm{ATV} / \mathrm{rtV}$ & 35 & 38 & & & & \\
\hline Case 8 & $1^{\text {st }}$ & Yes & No & $\mathrm{FTC}+\mathrm{TDF}+\mathrm{DRV} / \mathrm{rtV}$ & 1 & 38 & 450 & Undetectable & Yes & EC \\
\hline \multirow[t]{2}{*}{ Case 9} & $1^{\text {st }}$ & Yes & No & $\mathrm{ABC}+\mathrm{DDI}+\mathrm{LPV} / \mathrm{rtV}$ & 1 & 16 & 312 & 7653 & Yes & EC \\
\hline & & & & $\mathrm{AZT}+3 \mathrm{TC}+\mathrm{LPV} / \mathrm{rtV}$ & 17 & 36 & & & & \\
\hline \multirow[t]{2}{*}{ Case 10} & $1^{\text {st }}$ & Yes & Yes (from $6^{\text {th }}$ to $12^{\text {th }}$ week) & $\mathrm{FTC}+\mathrm{TDF}+\mathrm{EFV}$ & 1 & 5 & 240 & Undetectable & Yes & EC \\
\hline & & & & $\mathrm{AZT}+3 \mathrm{TC}+\mathrm{LPV} / \mathrm{rtV}$ & 13 & 38 & & & & \\
\hline Case 11 & $1^{\text {st }}$ & Unknown & No & $\mathrm{ABC}+\mathrm{TDF}+\mathrm{LPV} / \mathrm{rtV}$ & Unknown & 39 & Unknown & Undetectable & Yes & EC \\
\hline \multirow[t]{2}{*}{ Case 12} & $1^{\text {st }}$ & No & No & No & & & Unknown & Unknown & Yes & UC \\
\hline & $2^{\text {nd }}$ & Yes & No & $\mathrm{AZT}+3 \mathrm{TC}+\mathrm{LPV} / \mathrm{rtV}$ & 1 & 38 & 95 & Unknown & Yes & EC \\
\hline Case 13 & $1^{\text {st }}$ & Yes & No & $\mathrm{FTC}+\mathrm{NVP}$ & 1 & 39 & 673 & Undetectable & Yes & EC \\
\hline Case 14 & $1^{\text {st }}$ & Yes & No & $A Z T+3 T C+A B C$ & 1 & 35 & 564 & Undetectable & Yes & EC \\
\hline Case 15 & $1^{\text {st }}$ & Yes & No & $\mathrm{FTC}+\mathrm{TDF}+\mathrm{DRV} / \mathrm{rtV}$ & 1 & 37 & 501 & Undetectable & Yes & EC \\
\hline Case 16 & $1^{\text {st }}$ & No & No & $\mathrm{FTC}+\mathrm{TDF}+\mathrm{ATV} / \mathrm{rtV}$ & 16 & 36 & 522 & Undetectable & Yes & EC \\
\hline Case 17 & $1^{\text {st }}$ & No & No & $\mathrm{FTC}+\mathrm{TDF}+\mathrm{ATV}$ & 13 & 37 & 770 & Undetectable & Yes & EC \\
\hline \multirow[t]{2}{*}{ Case 18} & $1^{\text {st }}$ & Yes & No & $3 \mathrm{TC}$ & 1 & 28 & 440 & 13099 & Yes & LC \\
\hline & & & & $\mathrm{FTC}+\mathrm{TDF}+\mathrm{DRV} / \mathrm{rtV}$ & 29 & 36 & & & & \\
\hline Case 19 & $1^{\text {st }}$ & No & No & $\mathrm{FTC}+\mathrm{TDF}+\mathrm{ATV} / \mathrm{rtV}$ & 6 & 38 & 501 & Undetectable & Yes & EC \\
\hline \multirow[t]{2}{*}{ Case 20} & $1^{\text {st }}$ & No & No & $\mathrm{FTC}+\mathrm{TDF}+\mathrm{DRV} / \mathrm{rtV}+\mathrm{RAL}$ & 38 & 40 & 200 & Undetectable & Yes & EC \\
\hline & $2^{\text {nd }}$ & Unknown & Unknown & Unknown & & & 99 & Unknown & Unknown & Unknown \\
\hline
\end{tabular}


Table 2 Pregnancy features (Continued)

\begin{tabular}{|c|c|c|c|c|c|c|c|c|c|c|}
\hline$\overline{\text { Case } 21}$ & $1^{\mathrm{st}}$ & Yes & No & $\mathrm{FTC}+\mathrm{TDF}+\mathrm{ATV} / \mathrm{rtV}$ & 1 & 38 & 776 & 615 & Yes & $\overline{E C}$ \\
\hline Case 22 & $1^{\text {st }}$ & Yes & No & $\mathrm{AZT}+\mathrm{DDI}+\mathrm{LPV} / \mathrm{rtV}$ & 1 & 35 & 435 & Undetectable & Yes & EC \\
\hline \multirow[t]{2}{*}{ Case 23} & $1^{\text {st }}$ & Yes & Yes (from $3^{\text {rd }}$ to $9^{\text {th }}$ week) & $\mathrm{FTC}+\mathrm{TDF}+\mathrm{LPV} / \mathrm{rtv}$ & 1 & 3 & 275 & 20000 & Yes & EC \\
\hline & & & & $\mathrm{FTC}+\mathrm{TDF}+\mathrm{LPV} / \mathrm{rtV}$ & 10 & 38 & & & & \\
\hline
\end{tabular}

Legend. VL: viral load (copies/ml); AZT: zidovudine; 3TC: lamivudine; ABC: abacavir; FTC: emtricitabine; TDF: tenofovi; DDI: didanosine; NVP: nevirapine; LPV: lopinavir; ATV: atazanavir; FPV: fosamprenavir; DRV: darunavir; rtv: ritonavir booster; RAL: raltegravir; EC = elective caesarean section; UC = unspecified caesarean section; LC = caesarean section after labour. 
Table 3 HIV-1 exposed children features

\begin{tabular}{|c|c|c|c|c|c|c|c|c|c|c|}
\hline N. & $\begin{array}{l}\text { Birth } \\
\text { order }\end{array}$ & $\begin{array}{c}\text { Year of } \\
\text { birth }\end{array}$ & Gender & $\begin{array}{l}\text { Gestational } \\
\text { age (weeks) }\end{array}$ & $\begin{array}{c}\text { Weight at } \\
\text { birth (grams) }\end{array}$ & & $\begin{array}{c}\text { ART } \\
\text { prophylaxis }\end{array}$ & $\begin{array}{l}\text { Duration of postnatal } \\
\text { ART prophylaxis (days) }\end{array}$ & $\begin{array}{l}\text { HIV-1 } \\
\text { status }\end{array}$ & $\begin{array}{c}\text { Other pathological } \\
\text { conditions }\end{array}$ \\
\hline Case 1 & $1^{\text {st }}$ & 2010 & $M$ & 37 & 2590 & & AZT & 42 & SR & \\
\hline \multirow[t]{2}{*}{ Case 2} & $1^{\text {st }}$ & 2007 & $\mathrm{~F}$ & 38 & 2550 & & AZT & 45 & $S R$ & \\
\hline & $2^{\text {nd }}$ & 2009 & M & 38 & 3240 & & AZT & 19 & $S R$ & \\
\hline Case 3 & $1^{\text {st }}$ & 2009 & $\mathrm{~F}$ & 38 & 3100 & & $A Z T$ & 28 & SR & \\
\hline Case 4 & $1^{\text {st }}$ & 2007 & $\mathrm{~F}$ & 38 & 2920 & & $A Z T$ & 36 & $S R$ & \\
\hline \multirow[t]{3}{*}{ Case 5} & $1^{\text {st }}$ & 2005 & $\mathrm{~F}$ & 34 & 1975 & & AZT & 42 & $S R$ & \\
\hline & $2^{\text {nd }}$ & 2007 & $\mathrm{~F}$ & 37 & 1960 & SGA & $A Z T$ & 42 & $S R$ & HCV infection \\
\hline & $3^{\text {th }}$ & 2008 & $\mathrm{~F}$ & Unknown & Unknown & & AZT & 28 & $S R$ & \\
\hline \multirow[t]{2}{*}{ Case 6} & $1^{\text {st }}$ & 2001 & $\mathrm{~F}$ & 36 & 1880 & SGA & AZT & 44 & $S R$ & \\
\hline & $2^{\text {nd }}$ & 2002 & $\mathrm{~F}$ & 40 & 3080 & & AZT & 42 & $S R$ & \\
\hline Case 7 & $1^{\text {st }}$ & 2009 & M & 38 & 2530 & & AZT & 42 & $S R$ & \\
\hline Case 8 & $1^{\text {st }}$ & 2008 & $\mathrm{~F}$ & 38 & 2450 & & $A Z T$ & 43 & $S R$ & \\
\hline Case 9 & $1^{\text {st }}$ & 2008 & $\mathrm{~F}$ & 36 & 2520 & & AZT & 42 & $S R$ & \\
\hline Case 10 & $1^{\text {st }}$ & 2008 & M & 38 & 3250 & & AZT & 31 & SR & \\
\hline Case 11 & $1^{\text {st }}$ & 2008 & $\mathrm{~F}$ & 39 & 2770 & & $A Z T$ & 41 & SR & \\
\hline \multirow[t]{2}{*}{ Case 12} & $1^{\text {st }}$ & 2007 & M & 35 & 2270 & & $\mathrm{AZT}+3 \mathrm{TC}+\mathrm{NVP}$ & 31 & $S R$ & \\
\hline & $2^{\text {nd }}$ & 2008 & M & 38 & 3000 & & $\mathrm{AZT}+3 \mathrm{TC}+\mathrm{NVP}$ & 42 & $S R$ & \\
\hline Case 13 & $1^{\text {st }}$ & 2011 & M & 39 & 3100 & & $A Z T$ & 41 & $S R$ & \\
\hline Case 14 & $1^{\text {st }}$ & 2011 & $\mathrm{~F}$ & 35 & 1190 & SGA & AZT & 18 & $S R$ & \\
\hline Case 15 & $1^{\text {st }}$ & 2011 & M & 37 & 3190 & & AZT & 42 & $S R$ & \\
\hline Case 16 & $1^{\text {st }}$ & 2008 & $\mathrm{~F}$ & 36 & 2760 & & $A Z T$ & 42 & $S R$ & \\
\hline Case 17 & $1^{\mathrm{st}}$ & 2009 & M & 37 & 2520 & & AZT & 29 & $S R$ & Cryptorchidism \\
\hline Case 18 & $1^{\text {st }}$ & 2010 & M & 36 & 2180 & & AZT & 42 & $S R$ & \\
\hline Case 19 & $1^{\text {st }}$ & 2010 & $\mathrm{~F}$ & 38 & 2500 & & AZT & 14 & $S R$ & \\
\hline \multirow[t]{2}{*}{ Case 20} & $1^{\text {st }}$ & 2011 & $\mathrm{~F}$ & 40 & 2380 & SGA & $A Z T$ & 45 & $S R$ & \\
\hline & $2^{\text {nd }}$ & 2012 & $\mathrm{~F}$ & Unknown & Unknown & & Unknown & Unknown & $S R$ & \\
\hline Case 21 & $1^{\text {st }}$ & 2010 & $\mathrm{~F}$ & 38 & 2750 & & AZT & 42 & $S R$ & \\
\hline Case 22 & $1^{\text {st }}$ & 2012 & M & 35 & 2160 & & AZT & 42 & $S R$ & \\
\hline Case 23 & $1^{\text {st }}$ & 2011 & $M$ & 38 & 2690 & & $A Z T$ & 33 & $S R$ & \\
\hline
\end{tabular}

Legend. $\mathrm{M}=$ male; $\mathrm{F}=$ female; $\mathrm{AZT}=$ zidovudine; $3 T \mathrm{C}=$ lamivudine; $\mathrm{NVP}=$ nevirapine; $\mathrm{SR}=$ seroreverter; $\mathrm{SGA}=$ small for gestational age.

children born to HIV-1 perinatally-infected mothers described in this and other studies [3-11], only 2 acquired the infection, for a total MTCT rate of 1.5\%, comparable to that observed in the first-generation of exposed infants with the adoption of the same preventive measures, such as ART therapy during pregnancy, elective caesarean section, ART prophylaxis in the child, and bottlefeeding $[12,14,15]$.

Among 601 women with perinatally acquired HIV-1 infection enrolled in the ITLR, 383 are alive and over 16 years old at the last check. Thus, the 23 described in this case-series represent 6\% of those with a similar child-bearing age. It is worth noting that 5 had more than one child. In spite of the prolonged history of HIV1 infection and of targeted therapies, the good quality of life and a longer life expectancy presumably allowed these women to live motherhood with fewer distressing concerns. The low probability of HIV-1 transmission to the offspring is an additional important factor supporting such women having children. We have no documentation about the possible birth control methods adopted by these women, or whether these pregnancies were planned. However, the median age of our parturients was rather lower than the 32.3 years observed in the general Italian population [16]. This low median age at delivery is consistent with other reports $[3,5,6,10]$, and, perhaps, mirrors the fear that the chronic disease could deprive them of the special female role of motherhood. This growing desire of motherhood among seropositive women clearly emerges from our Register, where the 
number of HIV-1 perinatally-exposed children is around 350-400 per year, with an increasing multiparity rate, reaching $25 \%$ in recent years (data not shown).

In this scenario, the management of HIV-1 perinatallyinfected pregnant women and their children will be an important challenge for clinicians in the near future. A crucial aspect will be the choice of the best ART regimen to prevent MTCT. None of our mothers was perinatally exposed to ART drugs, as they were born before the ACTG 076 protocol $[17,18]$. However, the majority received ART therapy during childhood and experienced several sub-therapeutic regimens. Although resistance data were not available, these conditions are well known to favour the development of drug resistant viruses, with a consequent possible higher risk of MTCT, a phenomenon that fortunately does not emerge from the available data. As far as AZT is concerned, it is worth noting that over half of our mothers started AZT as a monotherapy during childhood, then shifted to a dual therapy including AZT, continuing with such combinations for several years. Six of these 14 women continued to receive AZT also during pregnancy, as did all their children as a prophylaxis, apparently with success, since the majority of the mothers had undetectable viral loads at delivery and no child acquired the infection. This casts doubts on the suggested exclusion of AZT during pregnancy if there had been a prolonged monotherapy during childhood [10]. Indeed, in a recent case series the role of intrapartum intravenous AZT for the prevention of MTCT in women with virological failure has been stressed [19]. AZT may be effective in the prevention of MTCT through other mechanisms besides its direct antiviral effect $[18,20]$.

The possible impact of a prolonged ART therapy on maternal and child health is a further important aspect. Even in this context, our results are reassuring. No congenital abnormalities were noticed, including the child exposed to EFV in the first 5 weeks of gestation [21,22]. Since maternal and neonatal complications due to ART drugs do not seem to be higher in perinatally-infected women, indications should not differ from those commonly recommended in seropositive mothers, for instance HAART should start as soon as possible [15], while stopping ART during pregnancy should be discouraged [12]. In fact, fearing a possible teratogenicity, 3 women discontinued ART treatment during the first trimester of pregnancy and their viral load was detectable at delivery. On the other hand, the experience of several therapeutic regimens during childhood and adolescence will presumably lead to the use of new antiretroviral drugs during pregnancy, such as integrase inhibitors, the impact of which on pregnant women and their foetuses still needs to be elucidated.

\section{Conclusions}

The outcome of second-generation HIV-1 exposed infants seems favourable. In spite of a long course of maternal infection, multiple ART regimens, and a possible development of drug-resistant viral strains, MTCT continues to be efficiently controlled by the commonly adopted preventive strategies, even though an adequate surveillance of pregnancy among this unique population is highly recommended.

\section{Competing interests}

The Italian Register for HIV Infection in Children was funded by the Istituto Superiore di Sanità, Rome, Italy, and the Paediatric European Network for Treatment of AIDS (PENTA) Foundation, Padua, Italy. The authors declare that they have no competing interests.

\section{Authors' contributions}

All Authors and Co-Authors contributed equally to the study design, acquisition of women and children information and interpretation of data. CC, CG and PAT drafted the manuscript, while MdM, LG, EC, CG, WB, OG, SE, SB critically revised it for its intellectual content. All Authors and Co-Authors accepted the final version of the manuscript.

\section{Authors' information}

Co-Authors: Giacomo Faldella (Bologna), Raffaele Badolato, Chiara Monfredini (Brescia), Cristina Gotta (Genoa), Vania Giacomet (Milan), Monica Cellini (Modena), Osvalda Rampon (Padua), Maura Agnese (Naples), Piero Valentini (Rome), Carlo Scolfaro, Silvia Garazzino (Turin), Antonio Mazza (Trento).

\section{Acknowledgements}

All principal investigators and sites who participate in the ITLR have been previously published [1] and are listed below. The Authors are grateful to Andrew Martin Garvey for editorial assistance and to Federica Messina for data management.

The "Italian Register for HIV infection in children" principal investigators by site are: Patrizia Osimani (Ancona), Domenico Larovere (Bari), Maurizio Ruggeri (Bergamo), Andrea Pession, Giacomo Faldella (Bologna), Francesca Capra, Sara Pulcini, Valentina Zattoni (Brescia), Maurizio Dedoni (Cagliari), Antonia Aliffi (Catania), Elisa Anastasio (Catanzaro), Elisa Fiumana (Ferrara), Paola Gervaso, Carlotta Montagnani (Florence), Antonio Di Biagio, Laura Ambra Nicolini, Laura De Hoffer, Maria Sole Acutis, Elisabetta Bondi (Genoa), Paola Erba, Valentina Fabiano, Giulia Ramponi, Filippo Salvini, Rita Lipreri, Susanna Esposito, Anna Plebani, Claudia Tagliabue (Milan), Francesca Giubbarelli (Modena), Emanuele Nicastro, Andrea Lo Vecchio, Wilma Buffolano, Maura Agnese (Naples), Amelia Romano (Palermo), Carlo Giaquinto, Osvalda Rampon, Martina Pennazzato (Padua), Rita Consolini (Pisa), Icilio Dodi (Parma), Anna Maccabruni (Pavia), Orazio Genovese, Paolo Palma, Giuseppe Pontrelli, Hyppolite Tchidjou (Rome), Paolina Olmeo (Sassari), Antonio Mazza (Trento), Erika Silvestro, Silvia Virano (Turin), Vincenzo Portelli (Trapani), Marco Rabusin (Trieste), Antonio Pellegatta (Varese).

\section{Author details}

${ }^{1}$ Department of Paediatrics, University of Turin, Turin, Italy. ${ }^{2}$ Department of Health Sciences, University of Florence, Florence, Italy. ${ }^{3}$ Department of Paediatrics, Padua University, Padua, Italy. ${ }^{4}$ Coordinating Centre for Perinatal Infection of Campania Region, Translational Medical Sciences Department of Federico II University, Naples, Italy. ${ }^{5}$ Department of Emergency, Catholic University of Rome, Rome, Italy. ${ }^{6}$ Paediatric Clinic 1, Department of Pathophysiology and Transplantation, Fondazione IRCCS Ca' Granda Ospedale Maggiore Policlinico, Milan, Italy. 'Department of Immunology and Infectious Diseases, "Bambino Gesù" Children's Hospital, Rome, Italy.

Received: 7 January 2014 Accepted: 9 April 2014

Published: 20 May 2014

\section{References}

1. De Martino M, Tovo PA, Balducci M, Galli L, Gabiano C, Rezza G, Pezzotti P: Reduction in mortality with availability of antiretroviral therapy for children with perinatal HIV-1 infection. Italian Register for HIV infection 
in children and the Italian National AIDS Registry. JAMA 2000, 284:190-197.

2. Gortmaker SL, Hughes M, Cervia J, Brady M, Johnson GM, Seage GR III, Song LY, Dankner WM, Oleske JM, Pediatric AIDS Clinical Trials Group Protocol 219 Team: Effect of combination therapy including protease inhibitors on mortality among children and adolescents infected with HIV-1. N Engl J Med 2001, 345:1522-1528.

3. Thorne C, Townsend CL, Peckham CS, Newell ML, Tookey PA: Pregnancies in young women with vertically acquired HIV infection in Europe. AIDS 2007, 21:2552-2556.

4. Elgalib A, Hegazi A, Samarawickrama A, Roedling S, Tariq S, Draeger E, Hemelaar J, Rathnayaka T, Azwa A, Hawkins D, Edwards S, Perez K, Russell J, Wood C, Poulton M, Shah R, Noble H, Rodgers M, Taylor GP, Anderson J, de Ruiter A: Pregnancy in HIV-infected teenagers in London. HIV Med 2011, 12:118-123.

5. Centers for Disease Control and Prevention: Pregnancy in perinatally HIVinfected adolescents and young adults-Puerto Rico, 2002. MMWR Morb Mortal Wkly Rep 2003, 52:149-151.

6. Chibber R, Khurranna A: Birth outcomes in perinatally HIV-infected adolescents and young adults in Manipur, India: a new frontier. Arch Gynecol Obstet 2005, 271:127-131.

7. Crane S, Sullivan M, Feingold M, Kaufman GE: Successful pregnancy in an adolescent with perinatally acquired human immunodeficiency virus. Obstet Gynecol 1998, 92:711.

8. Levine $A B$, Aaron E, Foster J: Pregnancy in perinatally HIV-infected adolescents. J Adolesc Health 2006, 38:765-768.

9. Brogly SB, Watts DH, Ylitalo N, Franco EL, Seage GR 3rd, Oleske J, Eagle M, Van Dyke R: Reproductive health of adolescent girls perinatally infected with HIV. Am J Public Health 2007, 97:1047-1052.

10. Williams SF, Keane-Tarchichi MH, Bettica L, Dieudonne A, Bardeguez AD: Pregnancy outcomes in young women with perinatally acquired human immunodeficiency virus-1. Am J Obstet Gynecol 2009, 200:149.e1-5.

11. Cruz MLS, Cardoso CA, João EC, Gomes IM, Abreu TF, Oliveira RH, Machado ES, Dias IR, Rubini NM, Succi RM: Pregnancy in HIV vertically infected adolescents and young women: a new generation of HIV-exposed infants. AIDS 2010, 24:2727-2731.

12. Galli L, Puliti D, Chiappini E, Gabiano C, Ferraris G, Mignone F, Viganò A, Giaquinto C, Genovese O, Anzidei G, Badolato R, Buffolano W, Maccabruni A, Salvini F, Cellini M, Ruggeri M, Manzionna M, Bernardi S, Tovo P, de Martino M, Italian Register for HIV Infection in Children: Is the interruption of antiretroviral treatment during pregnancy an additional major risk factor for mother-to-child transmission of HIV type 1? Clin Infect Dis 2009, 48:1310-1317.

13. Bertino E, Spada E, Occhi L, Coscia A, Giuliani F, Gagliardi L, Gilli G, Bona G, Fabris C, De Curtis M, Milani S: Neonatal anthropometric charts: the Italian neonatal study compared with other European studies. J Pediatr Gastroenterol Nutr 2010, 51:353-361.

14. Italian Register for Human Immunodeficiency Virus Infection in Children: Determinants of mother-to-infant human immunodeficiency virus 1 transmission before and after the introduction of zidovudine prophylaxis. Arch Pediatr Adolesc Med 2002, 156:915-921.

15. Centers for Disease Control and Prevention: Recommendations for Use of Antiretroviral Drugs in Pregnant HIV-1-Infected Women for Maternal Health and Interventions to reduce Perinatal HIV Transmission in the United States. 2012. Available at: http://aidsinfo.nih.gov/guidelines.

16. Italian Department of Health. Certificato di assistenza al parto (CeDAP). Analisi dell'evento nascita - Anno2009. Available at: http://www.salute.gov. it/imgs/C_17_pubblicazioni_1731_allegato.pdf.

17. Centers for Disease Control and Prevention: Zidovudine for the prevention of HIV transmission from mother to infant. MMWR Morb Mortal Wkly Rep 1994, 43:285-287.

18. Sperling RS, Shapiro DE, Coombs RW, Todd JA, Herman SA, McSherry GD, O'Sullivan MJ, Van Dyke RB, Jimenez E, Rouzioux C, Flynn PM, Sullivan JL: Maternal viral load, zidovudine treatment, and the risk of transmission of human immunodeficiency virus type 1 from mother to infant. Pediatric AIDS Clinical Trials Group Protocol 076 Study Group. N Engl I Med 1996, 335:1621-1629.

19. Briand N, Warszawski J, Mandelbrot L, Dollfus C, Pannier E, Cravello L, Nguyen R, Matheron I, Winer N, Tubiana R, Rouzioux C, Faye A, Blanche S, ANRS-EPF CO1-CO11 Study Group: Is intrapartum intravenous zidovudine for prevention of mother-to-child HIV-1 transmission still useful in the combination antiretroviral therapy era? Clin Infect Dis 2013, 57:903-914.

20. Bassetti D, Cargnel A: Genotypic resistance tests for the management of the HIV-infected pregnant woman. Scand I Infect Dis Suppl 2003, 106:70-74.

21. Ford N, Mofenson L, Kranzer K, Medu L, Frigati L, Mills EJ, Calmy A: Safety of efavirenz in first-trimester of pregnancy: a systematic review and meta-analysis of outcomes from observational cohorts. AIDS 2010 24:1461-1470

22. Ford N, Calmy A, Mofenson L: Safety of efavirenz in the first trimester of pregnancy: an updated systematic review and meta-analysis. AIDS 2011 25:2301-2304

doi:10.1186/1471-2334-14-277

Cite this article as: Calitri et al:: The second generation of HIV-1 vertically exposed infants: a case series from the Italian Register for paediatric HIV infection. BMC Infectious Diseases 2014 14:277.

\section{Submit your next manuscript to BioMed Central and take full advantage of:}

- Convenient online submission

- Thorough peer review

- No space constraints or color figure charges

- Immediate publication on acceptance

- Inclusion in PubMed, CAS, Scopus and Google Scholar

- Research which is freely available for redistribution 